\title{
RESPONSE OF THERMOELASTIC MICROPOLAR CUBIC CRYSTAL UNDER DYNAMIC LOAD AT AN INTERFACE
}

\author{
P. AILAWALIA* \\ Department of Applied Sciences and Humanities \\ Maharishi Markandeshwar University \\ Sadopur, Ambala City, Haryana (INDIA) \\ E-mail: praveen_2117@rediffmail.com \\ S.K. SACHDEVA \\ Department of Applied Sciences \\ D.A.V Institute of Engineering and Technology, Kabir \\ Nagar, Jalandhar, Punjab (INDIA) \\ (Research Scholar, Punjab Technical University, Jalandhar, Punjab, INDIA) \\ E-mail: sunilsachdeva.daviet@gmail.com \\ D. PATHANIA \\ Department of Applied Sciences, Guru Nanak DevJi Engineering College \\ Ludhiana, Punjab (INDIA) \\ E-mail: despathania@yahoo.com
}

\begin{abstract}
The purpose of this paper is to study the two dimensional deformation in a thermoelastic micropolar solid with cubic symmetry. A mechanical force is applied along the interface of a thermoelastic micropolar solid with cubic symmetry (Medium I) and a thermoelastic solid with microtemperatures (Medium II). The normal mode analysis has been applied to obtain the exact expressions for components of normal displacement, temperature distribution, normal force stress and tangential coupled stress for a thermoelastic micropolar solid with cubic symmetry. The effects of anisotropy, micropolarity and thermoelasticity on the above components have been depicted graphically.
\end{abstract}

Key words: thermoelasticity, cubic symmetry, microtemperature, normal mode.

\section{Introduction}

A micropolar continum is a collection of inter-connected particles in the form of small rigid bodies. The deformation in such materials is characterized by both translational and rotational motion. In this motion, the force at a point of the surface element of the body is completely determined by the stress vector at that point. Micropolar solids may represent the materials that are made up of dipole atoms and are subjected to surface and body couples. Polymeric materials, rocks, wood and fibre glass are few examples of such materials. Eringen and Suhubi [1] and Suhubi and Eringen [2] developed a non linear theory of microelastic solids. Later Eringen [3-5] developed a theory for the special class of micro-elastic materials and called it the "linear theory of micropolar elasticity". Under this theory, solids can undergo macrodeformations and micro-rotations. Materials affected by micromotions and microdeformations are known as micromorphic materials. Thermoelasticity is the study of equilibrium of bodies, treated as thermodynamic

\footnotetext{
* To whom correspondence should be addressed
} 
systems, whose interactions with the surroundings are restricted to the mechanical work, heat exchange and external forces. The change of body temperature is caused not only by the external and internal heat sources but also by the process of deformation itself. The micropolar theory was extended to include thermal effects by Nowacki [6], Eringen [7], Tauchert et al. [8], Tauchert [9], Nowacki and Olszak [10]. One can refer to Dhaliwal and Singh [11-12] for a review on the micropolar thermoelasticity, as well as to Eringen and Kafadar [13] in "Continuum Physics" series in which the general theory of micromorphic media has been summed up.

In the cubic symmetry, the materials have nine planes of symmetry whose normals are on the three coordinate axes and on the coordinate planes making an angle $\pi / 4$ with the coordinate axes. With the chosen coordinate system along the crystalline directions, the mechanical behavior of a cubic crystal can be characterized by four independent elastic constants $A_{1}, A_{2}, A_{3}$ and $A_{4}$. A wide class of crystals such as $\mathrm{Si}$, $\mathrm{Cu}, \mathrm{Ni}, \mathrm{Fe}, \mathrm{Au}, \mathrm{Al}$ etc., which are some frequently used substances, belong to cubic materials. Minagawa and Arakawa [14] discussed dispersion curves for waves in a cubic micropolar medium with reference to estimations of the material constants for diamond. Kumar and Ailawalia [15-18] and Ailawalia and Kumar [19] studied some source problems in a micropolar thermoelastic medium possessing cubic symmetry. Othman et al. [20] presented the effect of inclined load in a micropolar thermoelastic medium possessing cubic symmetry under three theories. Kumar and Partap [21] discussed the elastodynamic behavior of axisymmetric vibrations in a homogeneous isotropic micropolar thermoelastic cubic crystal plate. Lotfy and Yania [22] investigated the effect of the magnetic field and mode I crack in a micropolar thermoelastic cubic medium. Abbas et al. [23] studied the thermoelastic interaction in a thermally conducting cubic crystal subjected to ramp-type heating.

Grot [24] discussed a theory of thermodynamics of elastic bodies with microstructure whose microelements possess microtemperatures. Riha [25] studied heat conduction in materials with microtemperatures. Iesan and Quintanilla [26] studied a theory of thermoelasticity with microtemperatures. Iesan [27] proposed the theory of micromorphic elastic solids with microtemperatures. Exponential stability in thermoelasticity with microtemperatures was studied by Casas and Quintanilla [28]. Scalia and Svandze [29] gave the solutions of thermoelasticity with microtemperatures. Iesan [30] discussed thermoelasticity of bodies with microstructure and microtemperatures. Aouadi [31] discussed some theorems in the isotropic theory of microstretch thermoelasticity with microtemperatures. Quintanilla [32] discussed thermoelastic bodies with inner structure and microtemperatures. Scalia et al. [33] studied basic theorems in the equilibrium theory of thermoelasticity with microtemperatures. Quintanilla [34] discussed the growth and continuos dependence in thermoelasticity with microtemperatures. Steeb et al. [35] studied time harmonic waves in a thermoelastic material with microtemperatures. Chirita et al. [36] studied the theory of thermoelasticity with microtemperatures. Kumar and Kaur [37] studied the reflection and refraction of plane waves at the interface of an elastic solid and microstretch thermoelastic solid with microtemperatures. Ciarletta et al. [38] studied a homogeneous strongly elliptic thermoelastic medium with microstructures.

The present investigation is to determine the components of normal displacement, temperature distribution, normal force stress and tangential coupled stress in a thermoelastic micropolar solid with cubic symmetry due to mechanical source. The solution is obtained using normal mode analysis and effects of anisotropy, micropolarity and thermoelasticity on the above components are depicted graphically.

\section{Formulation of the problem}

We consider a normal force of magnitude $P_{l}$ acting along the interface of a micropolar thermoelastic cubic crystal (medium I) occupying the region $-\infty \leq y \leq 0$ and a thermoelastic medium with microtemperatures (medium II) in the region $0 \leq y \leq \infty$ as shown in Fig. 1.

We restrict our analysis to the plane strain parallel to the $x y$ plane with a displacement vector for micropolar thermoelastic solid with cubic symmetry (medium I) as $\boldsymbol{u}^{I}=\left(u_{1}^{I}, u_{2}^{I}, 0\right)$, microrotation vector as 
$\phi=\left(0,0, \phi_{3}\right)$ and displacement vector for a thermoelastic solid with microtemperatures (medium II) as $\boldsymbol{u}^{I I}=\left(u_{1}^{I I}, u_{2}^{I I}, 0\right)$ and micro-temperature vector as $\boldsymbol{w}^{I I}=\left(w_{1}^{I I}, w_{2}^{I I}, 0\right)$.

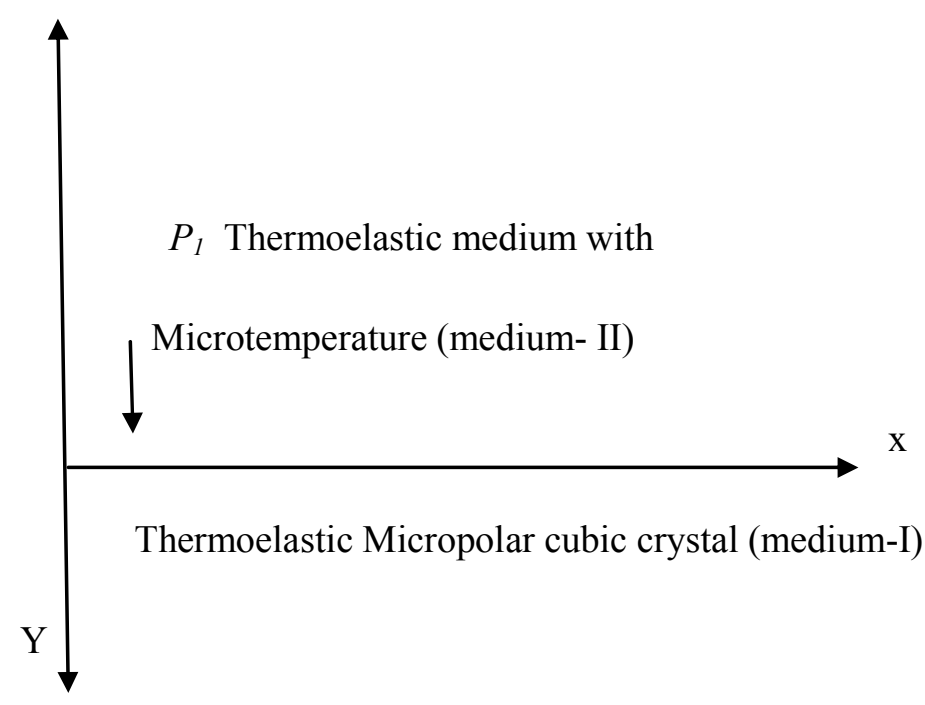

Fig.1. Geometry of the problem.

The field equations and constitutive relations in the absence of body forces, body couples and heat sources for medium I and medium II are given by:

For medium I, i.e., a micropolar thermoelastic medium with cubic symmetry, given by Kumar and Ailawalia $[15]$ as

$$
\begin{aligned}
& A_{1} \frac{\partial^{2} u_{1}^{I}}{\partial x^{2}}+\left(A_{2}+A_{4}\right) \frac{\partial^{2} u_{2}^{I}}{\partial x \partial y}+A_{3} \frac{\partial^{2} u_{1}^{I}}{\partial y^{2}}+\left(A_{3}-A_{4}\right) \frac{\partial \phi_{3}}{\partial y}-v_{1} \frac{\partial T_{1}}{\partial x}=\rho_{1} \frac{\partial^{2} u_{1}^{I}}{\partial t^{2}} \\
& A_{3} \frac{\partial^{2} u_{2}^{I}}{\partial x^{2}}+\left(A_{2}+A_{4}\right) \frac{\partial^{2} u_{1}^{I}}{\partial x \partial y}+A_{1} \frac{\partial^{2} u_{2}^{I}}{\partial y^{2}}-\left(A_{3}-A_{4}\right) \frac{\partial \phi_{3}}{\partial x}-v_{1} \frac{\partial T_{1}}{\partial y}=\rho_{1} \frac{\partial^{2} u_{2}^{I}}{\partial t^{2}} \\
& B_{3} \nabla^{2} \phi_{3}+\left(A_{3}-A_{4}\right)\left(\frac{\partial u_{2}^{I}}{\partial x}-\frac{\partial u_{1}^{I}}{\partial y}\right)-2\left(A_{3}-A_{4}\right) \phi_{3}=\rho_{1} j \frac{\partial^{2} \phi_{3}}{\partial t^{2}} \\
& K_{1}^{*} \nabla^{2} T_{1}=\rho_{1} c_{1}^{*} \frac{\partial T_{1}}{\partial t}+v_{1} T_{0} \frac{\partial}{\partial t}\left(\frac{\partial u_{1}^{I}}{\partial x}+\frac{\partial u_{2}^{I}}{\partial y}\right) \\
& \sigma_{y y}^{I}=A_{2} \frac{\partial u_{1}^{I}}{\partial x}+A_{1} \frac{\partial u_{2}^{I}}{\partial y}-v_{1} T_{1},
\end{aligned}
$$




$$
\begin{aligned}
& \sigma_{y x}^{I}=A_{4}\left(\frac{\partial u_{2}^{I}}{\partial x}-\phi_{3}\right)+A_{3}\left(\frac{\partial u_{1}^{I}}{\partial y}+\phi_{3}\right) \\
& m_{y z}=B_{3} \frac{\partial \phi_{3}}{\partial y} .
\end{aligned}
$$

For medium II, i.e., thermoelastic medium with microtemperatures, the equations are given by Steeb et al. $[35]$ as

$$
\begin{aligned}
& \left(\lambda_{2}+2 \mu_{2}\right) \frac{\partial^{2} u_{1}^{I I}}{\partial x^{2}}+\left(\lambda_{2}+\mu_{2}\right) \frac{\partial^{2} u_{2}^{I I}}{\partial x \partial y}+\mu_{2} \frac{\partial^{2} u_{1}^{I I}}{\partial y^{2}}-v_{2} \frac{\partial T_{2}}{\partial x}=\rho_{2} \frac{\partial^{2} u_{1}^{I I}}{\partial t^{2}} \\
& \left(\lambda_{2}+2 \mu_{2}\right) \frac{\partial^{2} u_{2}^{I I}}{\partial y^{2}}+\left(\lambda_{2}+\mu_{2}\right) \frac{\partial^{2} u_{1}^{I I}}{\partial x \partial y}+\mu_{2} \frac{\partial^{2} u_{2}^{I I}}{\partial x^{2}}-v_{2} \frac{\partial T_{2}}{\partial y}=\rho_{2} \frac{\partial^{2} u_{2}^{I I}}{\partial t^{2}} \\
& K_{2}^{*} \nabla^{2} T_{2}-a_{1} T_{0} \frac{\partial T_{2}}{\partial t}-v_{2} T_{0}\left(\frac{\partial u_{1}^{I I}}{\partial x}+\frac{\partial u_{2}^{I I}}{\partial y}\right)+k_{1}\left(\frac{\partial w_{1}^{I I}}{\partial x}+\frac{\partial w_{2}^{I I}}{\partial y}\right)=0 \\
& \left(k_{4}+k_{5}+k_{6}\right) \frac{\partial^{2} w_{1}^{I I}}{\partial x^{2}}+\left(k_{4}+k_{5}\right) \frac{\partial^{2} w_{2}^{I I}}{\partial x \partial y}+k_{6} \frac{\partial^{2} w_{1}^{I I}}{\partial y^{2}}-b \frac{\partial w_{1}^{I I}}{\partial t}-k_{2} w_{1}^{I I}-k_{3} \frac{\partial T_{2}}{\partial x}=0 \\
& k_{6} \frac{\partial^{2} w_{2}^{I I}}{\partial x^{2}}+\left(k_{4}+k_{5}\right) \frac{\partial^{2} w_{1}^{I I}}{\partial x \partial y}+\left(k_{4}+k_{5}+k_{6}\right) \frac{\partial^{2} w_{2}^{I I}}{\partial y^{2}}-b \frac{\partial w_{2}^{I I}}{\partial t}-k_{2} w_{2}^{I I}-k_{3} \frac{\partial T_{2}}{\partial y}=0 \\
& \sigma_{y y}^{I I}=\lambda_{2} \frac{\partial u_{1}^{I I}}{\partial x}+\left(\lambda_{2}+2 \mu_{2}\right) \frac{\partial u_{2}^{I I}}{\partial y}-v_{2} T_{2}, \\
& q_{y x}^{I I}=-k_{5} \frac{\partial w_{2}^{I I}}{\partial x}-k_{6} \frac{\partial w_{1}^{I I}}{\partial y} . \\
& \sigma_{y x}^{I I}=\mu_{2}\left(\frac{\partial u_{1}^{I I}}{\partial y}+\frac{\partial u_{2}^{I I}}{\partial x}\right), \\
& \partial w_{1}^{I I} \\
& \partial x
\end{aligned}
$$

For convenience the following non-dimensional variables are used

$$
x^{\prime}=\frac{1}{L} x, \quad y^{\prime}=\frac{1}{L} y, \quad u_{i}^{I^{\prime}}=\frac{1}{L} u_{i}^{I}, \quad u_{i}^{I I^{\prime}}=\frac{1}{L} u_{i}^{I I}, \quad w_{i}^{I I^{\prime}}=L w_{i}^{I I}, \quad t^{\prime}=\frac{c_{1}}{L} t,
$$




$$
\sigma_{i j}^{I^{\prime}}=\frac{\sigma_{i j}^{I}}{v_{2} T_{0}}, \quad \sigma_{i j}^{I{ }^{\prime}}=\frac{\sigma_{i j}^{I I}}{v_{2} T_{0}}, \quad \varphi_{3}^{\prime}=\varphi_{3}, \quad m_{i j}^{\prime}=\frac{m_{i j}}{L v_{2} T_{0}}, \quad q_{i j}^{I I^{\prime}}=\frac{q_{i j}^{I I}}{L c_{1} v_{2} T_{0}}, \quad T_{i}^{\prime}=\frac{T_{i}}{T_{0}}
$$

where $L$ is the standard length and $c_{1}$ is the longitudinal wave velocity in medium II given by $c_{1}^{2}=\frac{\lambda_{2}+2 \mu_{2}}{\rho_{2}}$.

Using the above non dimensional variables in Eqs (2.1)-(2.7), it may reduce these equations to (after dropping superscripts)

$$
\begin{aligned}
& d_{1} \frac{\partial^{2} u_{1}^{I}}{\partial x^{2}}+d_{2} \frac{\partial^{2} u_{2}^{I}}{\partial x \partial y}+d_{3} \frac{\partial^{2} u_{1}^{I}}{\partial y^{2}}+d_{4} \frac{\partial \phi_{3}}{\partial y}-d_{5} \frac{\partial T_{1}}{\partial x}=\frac{\partial^{2} u_{1}^{I}}{\partial t^{2}} \\
& d_{3} \frac{\partial^{2} u_{2}^{I}}{\partial x^{2}}+d_{2} \frac{\partial^{2} u_{1}^{I}}{\partial x \partial y}+d_{1} \frac{\partial^{2} u_{2}^{I}}{\partial y^{2}}-d_{4} \frac{\partial \phi_{3}}{\partial x}-d_{5} \frac{\partial T_{1}}{\partial y}=\frac{\partial^{2} u_{2}^{I}}{\partial t^{2}} \\
& \left(\frac{\partial^{2} \phi_{3}}{\partial x^{2}}+\frac{\partial^{2} \phi_{3}}{\partial y^{2}}\right)+d_{6}\left(\frac{\partial u_{2}^{I}}{\partial x}-\frac{\partial u_{1}^{I}}{\partial y}\right)-d_{7} \phi_{3}=d_{8} \frac{\partial^{2} \phi_{3}}{\partial t^{2}} \\
& \left(\frac{\partial^{2} T_{1}}{\partial x^{2}}+\frac{\partial^{2} T_{1}}{\partial y^{2}}\right)-d_{9} \frac{\partial T_{1}}{\partial t}-d_{10} \frac{\partial}{\partial t}\left(\frac{\partial u_{1}^{I}}{\partial x}+\frac{\partial u_{2}^{I}}{\partial y}\right)=0 \\
& \sigma_{y y}^{I}=d_{11} \frac{\partial u_{1}^{I}}{\partial x}+d_{12} \frac{\partial u_{2}^{I}}{\partial y}-d_{13} T_{1}, \\
& \sigma_{y x}^{I}=d_{14} \frac{\partial u_{2}^{I}}{\partial x}+d_{15} \frac{\partial u_{1}^{I}}{\partial y}+d_{16} \phi_{3}, \\
& m_{y z}=d_{17} \frac{\partial \phi_{3}}{\partial y}
\end{aligned}
$$

where

$$
\begin{aligned}
& d_{1}=\frac{A_{1}}{\rho_{1} c_{1}^{2}}, \quad d_{2}=\frac{A_{2}+A_{4}}{\rho_{1} c_{1}^{2}}, \quad d_{3}=\frac{A_{3}}{\rho_{1} c_{1}^{2}}, \quad d_{4}=\frac{A_{3}-A_{4}}{\rho_{1} c_{1}^{2}}, \quad d_{5}=\frac{\mathrm{v}_{1} T_{0}}{\rho_{1} c_{1}^{2}}, \\
& d_{6}=\frac{\left(A_{3}-A_{4}\right) L}{B_{3}}, \quad d_{7}=\frac{2\left(A_{3}-A_{4}\right) L^{2}}{B_{3}}, \quad d_{8}=\frac{\rho_{1} j c_{1}^{2}}{B_{3}}, \quad d_{9}=\frac{\rho_{1} c_{1}^{*} L c_{1}}{K_{1}^{*}}, \\
& d_{10}=\frac{\mathrm{v}_{1} L c_{1}}{K_{1}^{*}}, \quad d_{11}=\frac{A_{2}}{\mathrm{v}_{2} T_{0}}, \quad d_{12}=\frac{A_{1}}{\mathrm{v}_{2} T_{0}}, \quad d_{13}=\frac{\mathrm{v}_{1}}{\mathrm{v}_{2}}, \quad d_{14}=\frac{A_{4}}{\mathrm{v}_{2} T_{0}},
\end{aligned}
$$




$$
d_{15}=\frac{A_{3}}{\mathrm{v}_{2} T_{0}}, \quad d_{16}=\frac{\left(A_{3}-A_{4}\right)}{\mathrm{v}_{2} T_{0}}, \quad d_{17}=\frac{B_{3}}{L^{2} \mathrm{v}_{2} T_{0}}
$$

\section{Analytic solution}

The solution of the physical variable under consideration can be decomposed in terms of normal modes and can be considered in the following form

$$
\left(u_{i}^{I}, T_{i}, \varphi_{3}, \sigma_{i j}^{I}, m_{i j}, u_{i}^{I I}, w_{i}^{I I}, \sigma_{i j}^{I I}, q_{i j}^{I I}\right)(x, y, t)=\left(\bar{u}_{i}^{I}, \bar{T}_{i}, \bar{\varphi}_{3}, \bar{\sigma}_{i j}^{I}, \bar{m}_{i j}, \bar{u}_{i}^{I I}, \bar{w}_{i}^{I I}, \bar{\sigma}_{i j}^{I I}, \bar{q}_{i j}^{I I}\right)(y) e^{\omega t+i a x}
$$

where $\omega$ is the complex frequency, $a$ is the wave number in the $y$-direction and $\bar{u}_{i}^{I}(y), \bar{T}_{i}(y), \bar{\varphi}_{3}(y)$, $\bar{\sigma}_{i j}^{I}(y), \bar{m}_{i j}(y), \bar{u}_{i}^{I I}(y), \bar{w}_{i}^{I I}(y), \bar{\sigma}_{i j}^{I I}(y), \bar{q}_{i j}^{I I}(y)$ are the amplitudes of field quantities.

Using normal modes in Eqs (2.17)-(2.20), we get

$$
\begin{aligned}
& \left(d_{3} D^{2}-h_{42}\right) \bar{u}_{1}^{I}+h_{43} D \bar{u}_{2}^{I}+d_{4} D \bar{\phi}_{3}-h_{44} \bar{T}_{1}=0, \\
& h_{43} D \bar{u}_{1}^{I}+\left(d_{1} D^{2}-h_{45}\right) \bar{u}_{2}^{I}-h_{46} \bar{\phi}_{3}-d_{5} D \bar{T}_{1}=0 \\
& -d_{6} D \bar{u}_{1}^{I}+h_{47} \bar{u}_{2}^{I}+\left(D^{2}-h_{48}\right) \bar{\phi}_{3}=0 \\
& -h_{49} \bar{u}_{1}^{I}-h_{50} D \bar{u}_{2}^{I}+\left(D^{2}-h_{51}\right) \bar{T}_{1}=0
\end{aligned}
$$

where

$$
\begin{aligned}
& h_{42}=a^{2} d_{1}+\omega^{2}, \quad h_{43}=i a d_{2}, \quad h_{44}=i a d_{5}, \quad h_{45}=a^{2} d_{3}+\omega^{2}, \quad h_{46}=i a d_{4}, \\
& h_{47}=i a d_{6}, \quad h_{48}=a^{2}+d_{7}+d_{8} \omega^{2}, \quad h_{49}=i a \omega d_{10}, \quad h_{50}=\omega d_{10}, \quad h_{51}=a^{2}+d_{9} \omega .
\end{aligned}
$$

and constitutive relations (2.21)-(2.22) become

$$
\begin{aligned}
& \bar{\sigma}_{y y}=i a d_{11} \bar{u}_{1}^{I}+d_{12} D \bar{u}_{2}^{I}-d_{13} \bar{T}, \\
& \bar{\sigma}_{y x}=i a d_{14} \bar{u}_{2}^{I}+d_{15} D \bar{u}_{1}^{I}-d_{16} \bar{\phi}_{3}, \\
& \bar{m}_{y z}=d_{17} D \bar{\phi}_{3} .
\end{aligned}
$$

Eliminating $\bar{u}_{2}^{I}(y), \bar{\phi}_{3}(y), \bar{T}_{1}(y)$ between Eqs (3.1)-(3.2), we get the following eight order differential equation for $\bar{u}_{l}^{I}(y)$ as 


$$
\left(D^{8}-P D^{6}+Q D^{4}-R D^{2}+S\right) \bar{u}_{l}^{I}(y)=0
$$

where

$$
\begin{aligned}
& P=\frac{1}{d_{1} d_{3}}\left[d_{1} d_{3}\left(h_{48}+h_{51}\right)+\left(d_{3} h_{45}+d_{1} h_{42}\right)+d_{5} d_{3} h_{50}+h_{43}^{2}-d_{1} d_{4} d_{6}\right], \\
& Q=\frac{1}{d_{1} d_{3}}\left[d_{1} d_{3} h_{48} h_{51}+\left(d_{3} h_{45}+d_{1} h_{42}\right)\left(h_{48}+h_{51}\right)+h_{42} h_{45}+h_{46} h_{47} d_{3}+\right. \\
& +d_{5} h_{50}\left(d_{3} h_{48}-h_{42}\right)+h_{43}^{2}\left(h_{48}+h_{51}\right)+h_{43} h_{46} d_{6}+h_{43} h_{49} d_{5}+h_{43} h_{47} d_{4}+ \\
& \left.-d_{4} d_{6}\left(d_{1} h_{51}+d_{6} h_{45}\right)-d_{4} d_{5} d_{6} h_{50}+h_{43} h_{44} h_{50}+h_{44} h_{49} d_{1}\right], \\
& R=\frac{1}{d_{1} d_{3}}\left[h_{48} h_{51}\left(d_{3} h_{45}+d_{1} h_{42}\right)+h_{42} h_{45}\left(h_{48}+h_{51}\right)+h_{46} h_{47}\left(d_{3} h_{51}+h_{42}\right)+d_{5} h_{42} h_{50} h_{48}+\right. \\
& +h_{43}^{2} h_{48} h_{51}+h_{43} h_{51} h_{46} d_{6}+h_{43} h_{49} h_{48} d_{5}+h_{43} h_{47} h_{51} d_{4}-h_{45} h_{51} d_{4} d_{6}+ \\
& \left.+h_{47} h_{49} d_{4} d_{5}+h_{43} h_{44} h_{48} h_{50}+h_{44} h_{49}\left(d_{1} h_{48}+h_{45}\right)+h_{44} h_{46} h_{50} d_{6}\right], \\
& S=\frac{1}{d_{1} d_{3}}\left[h_{42} h_{51}\left(h_{45} h_{48}+h_{46} h_{47}\right)+h_{44} h_{45} h_{48} h_{49}-h_{44} h_{46} h_{47} h_{49}\right] .
\end{aligned}
$$

In a similar manner we can show that $\bar{u}_{2}^{I}(y), \bar{\phi}_{3}(y), \bar{T}_{1}(y)$ satisfies the equation

$$
\left(D^{8}-P D^{6}+Q D^{4}-R D^{2}+S\right)\left(\bar{u}_{2}^{I}(y), \bar{\phi}_{3}(y), \bar{T}_{1}(y)\right)=0,
$$

which can be factorized as follows

$$
\left(D^{2}-l_{1}^{2}\right)\left(D^{2}-l_{2}^{2}\right)\left(D^{2}-l_{3}^{2}\right)\left(D^{2}-l_{4}^{2}\right) \bar{u}_{1}^{I}(y)=0
$$

where $l_{n}^{2} ;(n=1,2,3,4)$ are roots of Eq.(3.10).

The series solution of Eq.(3.10) has the form

$$
\begin{aligned}
& \bar{u}_{1}^{I}(y)=\sum_{n=1}^{4}\left[L_{n}(a, \omega) e^{-l_{n} y}\right], \\
& \bar{u}_{2}^{I}(y)=\sum_{n=1}^{4}\left[L_{n}^{\prime}(a, \omega) e^{-l_{n} y}\right], \\
& \bar{T}_{1}(y)=\sum_{n=1}^{4}\left[L_{n}^{\prime \prime}(a, \omega) e^{-l_{n} y}\right],
\end{aligned}
$$




$$
\bar{\phi}_{3}(y)=\sum_{n=1}^{4}\left[L^{\prime \prime \prime}(a, \omega) e^{-l_{n} y}\right]
$$

where $L_{n}(a, \omega), L_{n}^{\prime}(a, \omega), L_{n}^{\prime \prime}(a, \omega)$ and $L_{n}^{\prime \prime \prime}(a, \omega)$ are specific functions depending upon $a, \omega$.

Using Eqs (3.11)-(3.14) in Eqs (3.1)-(3.4), we get the following relations

$$
\begin{aligned}
& L_{n}^{\prime}(a, \omega)=R_{1 n} L_{n}(a, \omega), \\
& L_{n}^{\prime \prime}(a, \omega)=R_{2 n} L_{n}(a, \omega), \\
& L_{n}^{\prime \prime \prime}(a, \omega)=R_{3 n} L_{n}(a, \omega) .
\end{aligned}
$$

Thus we have

$$
\begin{aligned}
& \bar{u}_{2}^{I}(y)=\sum_{n=1}^{4}\left[R_{1 n} L_{n}(a, \omega) e^{-l_{n} y}\right], \\
& \bar{T}_{1}(y)=\sum_{n=1}^{4}\left[R_{2 n} L_{n}(a, \omega) e^{-l_{n} y}\right], \\
& \bar{\phi}_{3}(y)=\sum_{n=1}^{4}\left[R_{3 n} L_{n}(a, \omega) e^{-l_{n} y}\right], \\
& \bar{\sigma}_{y y}(y)=\sum_{n=1}^{4}\left[R_{4 n} L_{n}(a, \omega) e^{-l_{n} y}\right], \\
& \bar{\sigma}_{y x}(y)=\sum_{n=1}^{4}\left[R_{5 n} L_{n}(a, \omega) e^{-l_{n} y}\right], \\
& \bar{m}_{y z}(y)=\sum_{n=1}^{4}\left[R_{6 n} L_{n}(a, \omega) e^{-l_{n} y}\right]
\end{aligned}
$$

where

$$
\begin{aligned}
& R_{l n}=-\frac{\left[-d_{3} d_{5} l_{n}^{5}+\left(d_{5} h_{42}+h_{43} h_{44}+h_{48} d_{3} d_{5}-d_{4} d_{5} d_{6}\right) l_{n}^{3}-\left\{h_{48}\left(d_{5} h_{42}+h_{43} h_{44}\right)-d_{6} h_{44} h_{46}\right\} l_{n}\right]}{\left[\left(d_{5} h_{43}-d_{1} h_{44}\right) l_{n}^{4}+\left\{h_{44} h_{45}-h_{48}\left(d_{5} h_{43}-d_{1} h_{44}\right)-h_{47} d_{4} d_{5}\right\} l_{n}^{2}-h_{44}\left(h_{45} h_{48}-h_{46} h_{47}\right)\right]}, \\
& R_{2 n}=\frac{\left[h_{49}+h_{50} l_{n} R_{1 n}\right]}{\left[l_{n}^{2}-h_{51}\right]},
\end{aligned}
$$




$$
\begin{aligned}
& R_{3 n}=-\frac{\left[d_{6} l_{n}+h_{47} R_{1 n}\right]}{\left[l_{n}^{2}-h_{48}\right]}, \\
& R_{4 n}=\left[i a d_{11}-l_{n} d_{12} R_{1 n}-d_{13} R_{2 n}\right], \\
& R_{5 n}=\left[\mathrm{iad}_{14} R_{1 n}-l_{n} d_{15}+d_{16} R_{3 n}\right], \\
& R_{6 n}=\left[-l_{n} d_{17} R_{3 n}\right] .
\end{aligned}
$$

Adopting the same methodology, the solutions for medium II (i.e., a thermoelastic solid with microtemperatures), are of the form

$$
\begin{aligned}
& \bar{u}_{1}^{I I}(y)=\sum_{m=1}^{5}\left[M_{m}(a, \omega) e^{-r_{m} y}\right], \\
& \bar{u}_{2}^{I I}(y)=\sum_{m=1}^{5}\left[M_{m}^{\prime}(a, \omega) e^{-r_{m} y}\right], \\
& \bar{T}_{2}(y)=\sum_{m=1}^{5}\left[M_{m}^{\prime \prime}(a, \omega) e^{-r_{m} y}\right], \\
& \bar{w}_{1}^{I I}(y)=\sum_{m=1}^{5}\left[M_{m}^{\prime \prime}(a, \omega) e^{-r_{m} y}\right], \\
& \bar{w}_{2}^{I I}(y)=\sum_{m=1}^{5}\left[M_{m}^{\prime \prime \prime}(a, \omega) e^{-r_{m} y}\right]
\end{aligned}
$$

where $r_{m}^{2} ;(m=1,2,3,4,5)$ are the roots of the characteristic equation,

$$
\left(D^{10}+A D^{8}+B D^{6}+C D^{4}+E D^{2}+F\right) \bar{u}_{1}^{I I}(y)=0
$$

and

$$
\begin{aligned}
& A=\frac{1}{h_{13}^{2} h_{18} h_{20}}\left[-h_{13}\left\{h_{13}\left(h_{20} h_{35}+h_{18} h_{34}+h_{40}^{2}+h_{18} h_{20} h_{33}-h_{17} h_{20}\right)+\right.\right. \\
& \left.\left.+h_{18} h_{20} h_{32}+h_{14} h_{16} h_{20} h_{18}\right\}-h_{18} h_{20} h_{13} h_{31}+h_{20} h_{18} h_{36}^{2}\right],
\end{aligned}
$$




$$
\begin{aligned}
& B=\frac{1}{h_{13}^{2} h_{18} h_{20}}\left[h _ { 1 3 } \left\{h _ { 1 3 } \left(h_{34} h_{35}+h_{20} h_{33} h_{35}+h_{18} h_{33} h_{34}+h_{33} h_{40}^{2}+h_{17} h_{40} h_{41}+\right.\right.\right. \\
& \left.\left.-h_{17} h_{34}-h_{18} h_{39} h_{41}-h_{39} h_{40}\right)+h_{32}\left(h_{20} h_{35}+h_{18} h_{34}+h_{40}^{2}+h_{18} h_{33} h_{20}-h_{17} h_{20}\right)\right\}+ \\
& +h_{13}\left\{h_{14} h_{16}\left(h_{20} h_{35}+h_{18} h_{34}+h_{40}^{2}\right)\right\}+h_{31}\left\{h_{13}\left(h_{20} h_{35}+h_{18} h_{34}+h_{40}^{2}+h_{18} h_{33} h_{20}-h_{17} h_{20}\right)+\right. \\
& \left.+h_{18} h_{20} h_{32}+h_{20} h_{14} h_{18} h_{16}\right\}-\left\{h_{14} h_{18} h_{20} h_{36} h_{38}+\right. \\
& \left.\left.-h_{36}^{2}\left(h_{18} h_{34}+h_{35} h_{20}+h_{40}^{2}+h_{18} h_{33} h_{20}-h_{17} h_{20}\right)\right\}+h_{18} h_{20} h_{13} h_{37} h_{38}\right] \text {, } \\
& C=\frac{1}{h_{13}^{2} h_{18} h_{20}}\left[h _ { 1 3 } \left\{h_{13} h_{35}\left(h_{39} h_{41}-h_{33} h_{34}\right)-h_{32}\left(h_{35} h_{34}+h_{20} h_{33} h_{35}+h_{18} h_{33} h_{34}+\right.\right.\right. \\
& \left.\left.+h_{33} h_{40}^{2}+h_{17} h_{40} h_{41}-h_{17} h_{34}-h_{18} h_{39} h_{41}-h_{39} h_{40}\right)-h_{14} h_{16} h_{34} h_{35}\right\}+ \\
& -h_{31}\left\{h _ { 1 3 } \left(h_{34} h_{35}+h_{20} h_{33} h_{35}+h_{18} h_{33} h_{34}+h_{33} h_{40}^{2}+h_{17} h_{40} h_{41}-h_{17} h_{34}+\right.\right. \\
& \left.\left.-h_{18} h_{39} h_{41}-h_{39} h_{40}\right)+h_{32}\left(h_{20} h_{35}+h_{18} h_{34}+h_{40}^{2}+h_{18} h_{33} h_{20}-h_{17} h_{20}\right)\right\}+ \\
& -h_{31}\left\{h_{14} h_{16}\left(h_{20} h_{35}+h_{18} h_{34}+h_{40}^{2}\right)\right\}-h_{36}^{2}\left\{h_{35} h_{34}+h_{20} h_{33} h_{35}+h_{18} h_{33} h_{34}+h_{33} h_{40}^{2}+\right. \\
& \left.-h_{39} h_{40}\left(h_{18}+1\right)+h_{17}\left(h_{40} h_{41}-h_{34}\right)\right\}+h_{14} h_{36} h_{38}\left(h_{20} h_{35}+h_{18} h_{34}+h_{40}^{2}\right)+ \\
& \left.-\left\{h_{18} h_{20} h_{32} h_{37} h_{38}+h_{13} h_{37} h_{38}\left(h_{20} h_{35}+h_{18} h_{34}+h_{40}^{2}\right)-h_{18} h_{20} h_{16} h_{37} h_{36}\right\}\right], \\
& E=\frac{1}{h_{13}^{2} h_{18} h_{20}}\left[-h_{32} h_{13} h_{35}\left(h_{39} h_{41}-h_{33} h_{34}\right)-h_{31}\left\{h_{35} h_{13}\left(h_{39} h_{41}-h_{33} h_{34}\right)+\right.\right. \\
& -h_{32}\left(h_{34} h_{35}+h_{20} h_{33} h_{35}+h_{18} h_{34} h_{33}+h_{33} h_{40}^{2}+h_{17} h_{40} h_{41}-h_{17} h_{34}+\right. \\
& \left.\left.-h_{18} h_{39} h_{41}-h_{39} h_{40}\right)-h_{14} h_{16} h_{34} h_{35}\right\}-\left\{h_{36}^{2} h_{35}\left(h_{39} h_{41}-h_{33} h_{34}\right)+\right. \\
& \left.+h_{14} h_{36} h_{34} h_{35} h_{38}\right\}-\left\{h_{16} h_{36} h_{37}\left(h_{20} h_{35}+h_{18} h_{34}+h_{40}^{2}\right)+\right. \\
& \left.\left.-h_{13} h_{37} h_{34} h_{35} h_{38}-h_{32} h_{37} h_{38}\left(h_{20} h_{35}+h_{18} h_{34}+h_{40}^{2}\right)\right\}\right] \\
& F=\frac{1}{h_{13}^{2} h_{18} h_{20}}\left[h_{32} h_{31} h_{35}\left(h_{39} h_{41}-h_{33} h_{34}\right)-h_{34} h_{35} h_{37}\left(h_{32} h_{38}-h_{16} h_{36}\right)\right], \\
& h_{11}=\frac{\left(\lambda_{2}+2 \mu_{2}\right)}{\rho_{2} c_{1}^{2}}, \quad h_{12}=\frac{\left(\lambda_{2}+\mu_{2}\right)}{\rho_{2} c_{1}^{2}}, \quad h_{13}=\frac{\mu_{2}}{\rho_{2} c_{1}^{2}}, \quad h_{14}=\frac{\mathrm{v}_{2} T_{0}}{\rho_{2} c_{1}^{2}}, \quad h_{15}=\frac{a_{1} T_{0} c_{1} L}{K_{2}^{*}} \\
& h_{16}=\frac{v_{2} L^{2}}{K_{2}^{*}}, \quad h_{17}=\frac{k_{1}}{K_{2}^{*} T_{0}}, \quad h_{18}=\frac{k_{4}+k_{5}+k_{6}}{L^{2} k_{3} T_{0}}, \quad h_{19}=\frac{k_{4}+k_{5}}{L^{2} k_{3} T_{0}}, \quad h_{20}=\frac{k_{6}}{L^{2} k_{3} T_{0}} \\
& h_{21}=\frac{b c_{1}}{L k_{3} T_{0}}, \quad h_{22}=\frac{k_{2}}{k_{3} T_{0}}, \quad h_{23}=\frac{\left(\lambda_{2}+2 \mu_{2}\right)}{v_{2} T_{0}}, \quad h_{24}=\frac{\lambda_{2}}{v_{2} T_{0}}, \quad h_{25}=\frac{\mu_{2}}{v_{2} T_{0}},
\end{aligned}
$$




$$
\begin{aligned}
& h_{26}=\frac{\mu_{2}}{v_{2} T_{0}}, \quad h_{27}=\frac{k_{4}+k_{5}+k_{6}}{L^{3} c_{1} v_{2} T_{0}}, \quad h_{28}=\frac{k_{4}}{L^{3} c_{1} v_{2} T_{0}}, \quad h_{29}=\frac{k_{5}}{L^{3} c_{1} v_{2} T_{0}}, \quad h_{30}=\frac{k_{6}}{L^{3} c_{1} v_{2} T_{0}}, \\
& h_{31}=\omega^{2}+a^{2} h_{11}, \quad h_{32}=\omega^{2}+a^{2} h_{13}, \quad h_{33}=a^{2}+\omega h_{15}, \quad h_{34}=a^{2} h_{18}+\omega h_{21}+h_{22}, \\
& h_{35}=a^{2} h_{20}+\omega h_{21}+h_{22}, \quad h_{36}=i a h_{12}, \quad h_{37}=i a h_{14}, \quad h_{38}=-i a h_{16}, \quad h_{39}=i a h_{17}, \\
& h_{40}=i a h_{19}, \quad h_{41}=-i a .
\end{aligned}
$$

Thus Eqs (3.24)-(3.28) and constitutive relations in medium II may be expressed in the form

$$
\begin{aligned}
& \bar{u}_{2}^{I I}(y)=\sum_{m=1}^{5}\left[H_{1 m} M_{m}(a, \omega) e^{-r_{m} y}\right], \\
& \bar{T}_{2}(y)=\sum_{m=1}^{5}\left[H_{2 m} M_{m}(a, \omega) e^{-r_{m} y}\right], \\
& \bar{w}_{1}^{I I}(y)=\sum_{m=1}^{5}\left[H_{3 m} M_{m}(a, \omega) e^{-r_{m} y}\right], \\
& \bar{w}_{2}^{I I}(y)=\sum_{m=1}^{5}\left[H_{4 m} M_{m}(a, \omega) e^{-r_{m} y}\right], \\
& \bar{\sigma}_{y y}^{I I}(y)=\sum_{m=1}^{5}\left[H_{5 m} M_{m}(a, \omega) e^{-r_{m} y}\right], \\
& \bar{\sigma}_{y x}^{I I}(y)=\sum_{m=1}^{5}\left[H_{6 m} M_{m}(a, \omega) e^{-r_{m} y}\right], \\
& \bar{q}_{y x}^{I I}(y)=\sum_{m=1}^{5}\left[H_{8 m} M_{m}(a, \omega) e^{-r_{m} y}\right] \\
& \bar{q}_{y y}^{I I}(y)=\sum_{m=1}^{5}\left[h_{7 m} M_{m}(a, \omega) e^{-r_{m} y}\right], \\
& {\left[\left(h_{14} h_{36}-h_{13} h_{37}\right) r_{m}^{2}+h_{32} h_{37}\right]} \\
& \left.h_{13} r_{m}^{2}-h_{31}-i a h_{12} r_{m} H_{1 m}\right) \\
& h_{37}
\end{aligned}
$$

where 


$$
\begin{aligned}
& H_{3 m}=\frac{\left[h_{40} r_{m}^{2}-\left(h_{33} h_{40}+h_{17} h_{41}\right)\right] H_{2 m}+h_{16} h_{40} r_{m} H_{1 m}+h_{38} h_{40}}{\left[h_{17} h_{20} r_{m}^{2}-\left(h_{39} h_{40}+h_{34} h_{37}\right)\right]}, \\
& H_{4 m}=\frac{\left[H_{2 m}+h_{40} H_{3 m}\right] r_{m}}{\left[h_{35}-h_{18} r_{m}^{2}\right]} \\
& H_{5 m}=\left[i a h_{24}-r_{m} h_{23} H_{1 m}-H_{2 m}\right], \\
& H_{6 m}=\left[i a h_{25} H_{1 m}-r_{m} h_{26}\right], \\
& H_{7 m}=-i a h_{28} H_{3 m}+h_{27} r_{m} H_{4 m}, \\
& H_{8 m}=h_{30} r_{m} H_{3 m}-i a h_{29} H_{4 m} .
\end{aligned}
$$

\section{Applications}

In this section we determine the parameter $L_{n} ;(n=1,2,3,4)$ and $M_{m} ;(m=1,2,3,4,5)$. In the physical problem, we should suppress the positive exponential that are unbounded at infinity. Constants $L_{1}, L_{2}, L_{3}, L_{4}$ and $M_{1}, M_{2}, M_{3}, M_{4}, M_{5}$ have to be selected such that the boundary condition at the surface $y=0$ takes the form

$$
\begin{aligned}
& \sigma_{y y}^{I}=\sigma_{y y}^{I I}-P_{1} e^{\omega t+i a x} ; \quad \sigma_{y x}^{I}=\sigma_{y x}^{I I} ; \quad u_{1}^{I}=u_{1}^{I I} ; \quad u_{2}^{I}=u_{2}^{I I} ; \\
& m_{y z}=0 ; \quad K_{1}^{*} \frac{\partial T_{1}}{\partial y}=K_{2}^{*} \frac{\partial T_{2}}{\partial y} ; \quad q_{y y}^{I I}=0 ; \quad q_{y x}^{I I}=0 ; \quad T_{1}=T_{2}
\end{aligned}
$$

where $P_{1}$ is the magnitude of mechanical force.

Using the expressions for $\sigma_{y y}^{I}, \sigma_{y y}^{I I}, \sigma_{y x}^{I}, \sigma_{y x}^{I I}, u_{1}^{I}, u_{1}^{I I}, u_{2}^{I}, u_{2}^{I I}, m_{y z}, T_{1}, T_{2}$ qyy $q_{y x}^{I I}$ from Eqs (3.18)-(3.23) and (3.30)-(3.37) in the above boundary conditions, we obtain the following non homogenous linear equations as

$$
\begin{aligned}
& \sum_{n=1}^{4}\left[R_{4 n} L_{n}\right]-\sum_{m=1}^{5}\left[H_{5 m} M_{m}\right]=-P_{1} \\
& \sum_{n=1}^{4}\left[R_{5 n} L_{n}\right]-\sum_{m=1}^{5}\left[H_{6 m} M_{m}\right]=0 \\
& \sum_{n=1}^{4}\left[L_{n}\right]-\sum_{m=1}^{5}\left[M_{m}\right]=0 \\
& \sum_{n=1}^{4}\left[R_{1 n} L_{n}\right]-\sum_{m=1}^{5}\left[H_{1 m} M_{m}\right]=0
\end{aligned}
$$




$$
\begin{aligned}
& \sum_{n=1}^{4}\left[R_{6 n} L_{n}\right]=0 \\
& K_{1}^{*} \sum_{n=1}^{4}\left[l_{n} R_{2 n} L_{n}\right]-K_{2}^{*} \sum_{m=1}^{5}\left[r_{m} H_{2 m} M_{m}\right]=0, \\
& \sum_{m=1}^{5}\left[H_{7 m} M_{m}\right]=0 \\
& \sum_{m=1}^{5}\left[H_{8 m} M_{m}\right]=0 \\
& \sum_{n=1}^{4}\left[R_{2 n} L_{n}\right]-\sum_{m=1}^{5}\left[H_{2 m} M_{m}\right]=0 .
\end{aligned}
$$

After solving the above system of nine equations, we get the values of constants $L_{1}, L_{2}, L_{3}, L_{4}, M_{1}, M_{2}, M_{3}, M_{4}$ and $M_{5}$ and hence obtain the components of normal displacement, temperature distribution, normal force stress and tangential couple stress for a thermoelastic micropolar solid with cubic symmetry at the interface of a thermoelastic solid with microtemperatures.

\section{Special cases}

1) Substituting $A_{1}=\left(\lambda_{1}+2 \mu_{1}+k\right), A_{2}=\lambda_{1}, A_{3}=\left(\mu_{1}+k\right), A_{4}=\mu_{1}, B_{3}=\gamma$, we obtain the expression for the micropolar thermoelastic solid (MTS).

2) Neglecting the micropolarity effect, i.e., $B_{3}=j=0$ and $A_{3}=A_{4}$, the corrosponding expression are obtain in a thermoelastic solid with cubic symmetry (TCC).

3) Taking, $A_{1}=\left(\lambda_{1}+2 \mu_{1}\right), A_{2}=\lambda_{1}, A_{3}=\mu_{1}, A_{4}=\mu_{1}, B_{3}=0$ in the expression obtained in the previous step, the expressions for normal displacement, temperature distribution and normal force stress are obtained for thermoelastic solid(TS).

\section{Numerical results and discussions}

In order to illustrate the theoretical results obtained in the preceding section, we take the following values of parameters for the micropolar solid with cubic symmetry as [15]

$$
\begin{array}{ll}
A_{1}=19.6 \times 10^{10} \mathrm{~N} / \mathrm{m}^{2}, & A_{2}=5.6 \times 10^{10} \mathrm{~N} / \mathrm{m}^{2}, \quad A_{3}=11.7 \times 10^{10} \mathrm{~N} / \mathrm{m}^{2}, \\
A_{4}=11.7 \times 10^{10} \mathrm{~N} / \mathrm{m}^{2}, & B_{3}=0.98 \times 10^{-9} \mathrm{~N} .
\end{array}
$$

For micropolar thermoelastic solid, we take the following values of relevant parameters in case of Magnesium crystal like material as [15]

$$
\lambda_{1}=9.4 \times 10^{10} \mathrm{~N} / \mathrm{m}^{2}, \quad \mu_{1}=4.0 \times 10^{10} \mathrm{~N} / \mathrm{m}^{2}, \quad \rho_{1}=1.74 \times 10^{3} \mathrm{~kg} / \mathrm{m}^{3}, \quad k=10^{10} \mathrm{Nm}^{-2},
$$




$$
\begin{aligned}
& \gamma=0.779 \times 10^{-9} \mathrm{~N}, \quad j=0.0000002 \times 10^{-14} \mathrm{~m}^{2}, \quad c_{1}^{*}=0.104 \times 10^{4} \mathrm{Nm} / \mathrm{Kg} / \mathrm{K}, \\
& T_{0}=298 \mathrm{~K}, \quad K_{1}^{*}=1.7 \times 10^{2} \mathrm{Ns}^{-1} \mathrm{~K}^{-1}, \quad \mathrm{v}_{1}=0.0268 \times 10^{8} \mathrm{~N} / \mathrm{m}^{2} \mathrm{~K} .
\end{aligned}
$$

The values of relevant parameters for the thermoelastic solid with microtemperatures are [35]

$$
\begin{aligned}
& \lambda_{2}=2.17 \times 10^{10} \mathrm{~N} / \mathrm{m}^{2}, \quad \mu_{2}=3.278 \times 10^{10} \mathrm{~N} / \mathrm{m}^{2}, \quad \rho_{2}=1.74 \times 10^{3} \mathrm{~kg} / \mathrm{m}^{3}, \\
& b=1.389 \times 10^{10} \mathrm{~N}, \quad K_{2}^{*}=1.7 \times 10^{2} \mathrm{Ns}^{-1} \mathrm{~K}^{-1}, \quad a_{1} T_{0}=1.8 \times 10^{6} \mathrm{Jm}^{-3} \mathrm{~K}^{-1}, \\
& \mathrm{v}_{2}=0.0268 \times 10^{8} \mathrm{Nm}^{-2} \mathrm{~K}^{-1} ., \quad k_{1}=2 \times 10^{10} \mathrm{Wm}^{-1}, \quad k_{2}=0.1 \times 10^{10} \mathrm{Wm}^{-1}, \\
& k_{3}=0.4 \times 10^{10} \mathrm{Wm}^{-1} \mathrm{~K}^{-1}, \quad k_{4}=0.3 \times 10^{10} \mathrm{Wm}^{-3} \mathrm{~K}^{-1}, \quad k_{5}=0.5 \times 10^{10} \mathrm{Wm}^{-3} \mathrm{~K}^{-1}, \\
& k_{6}=0.7 \times 10^{10} \mathrm{Wm}^{-3}, \quad L=1.0 \times 10^{-10} \mathrm{~m} .
\end{aligned}
$$

The computations are carried out for the value of non-dimensional time $t=0.2$ in the range $0 \leq x \leq 10.0$ and on the surface $y=1.0$. The numerical values for normal displacement, temperature distribution, normal force stress and tangential coupled stress are shown in Figs 2-5 for mechanical force with magnitude

$$
P_{1}=1.0, \quad \omega=\omega_{0}+1 \xi, \quad \omega_{0}=-0.3, \quad \xi=0.1 \quad \text { and } \quad a=0.9 \quad \text { for }
$$

(a) Micropolar thermoelastic solid with cubic symmetry (MTCC) by solid line with centered symbol

(b) Thermoelastic solid with cubic symmetry (TCC) by solid line with centered symbol

(c) Micropolar thermoelastic solid (MTS) by dashed line with centered symbol

(d) Thermoelastic solid (MTS) by dashed line with centered symbol $\mathrm{x}$.

\section{Discussions}

The variations of normal displacement for MTCC, TCC and TS are similar in nature. The variation for MTS are opposite in nature as observed from Fig.2. It is also observed that the variations of normal displacement for MTCC and MTS are mirror images of each other. The variations of temperature distribution are quite similar in nature for the thermoelastic medium with cubic symmetry (MTCC and TCC) as well as for the thermoelastic medium without cubic symmetry (MTS and TS). These variations of temperature distributions are shown in Fig.3.

It can be observed form Fig. 4 that the variations of normal force stress are opposite in nature for the micropolar thermoelastic medium (MTCC and MTS). These values of normal force stress are less in magnitude for TCC. The values for all medium coincides at $x=3.0$ and $x=7.0$. The variations of tangential couple stress are exactly mirror images of each other as observed from Fig.5.

\section{Conclusion}

Anisotropy and micropolarity show a significant effect on all the quantities. The variations of temperature distribution are similar in nature for the anisotropic medium(MTCC and TCC) and isotropic 
medium(MTS and TS). Due to the anisotropic effect, the variations of normal force stress are opposite in nature for the micropolar thermoelastic medium (MTCC and MTS). The values of the quantities coincide for different media at $x=7.0$.

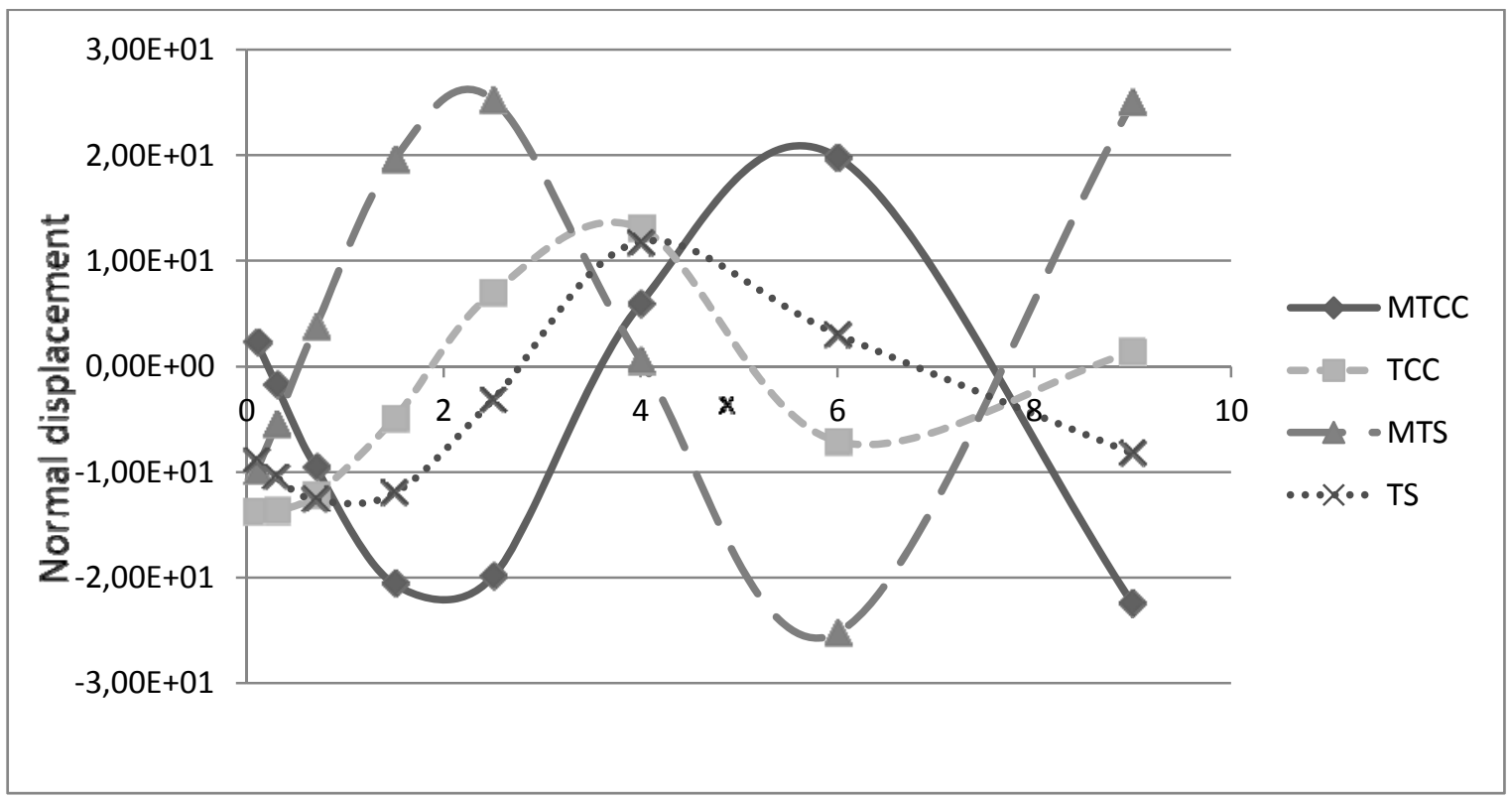

Fig.2. Variation of normal displacement with horizontal distance.

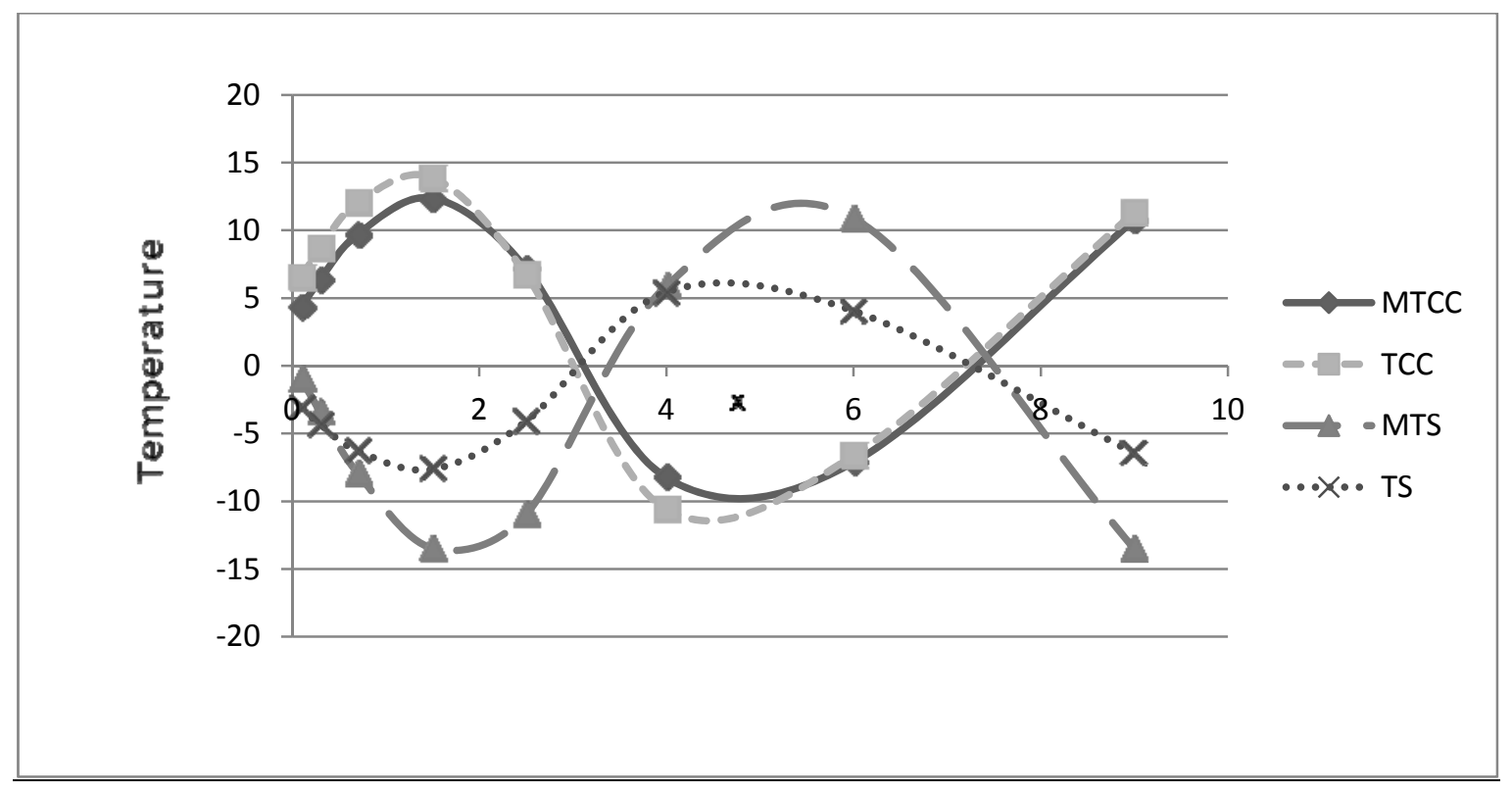

Fig.3. Variation of temperature distribution with horizontal distance. 


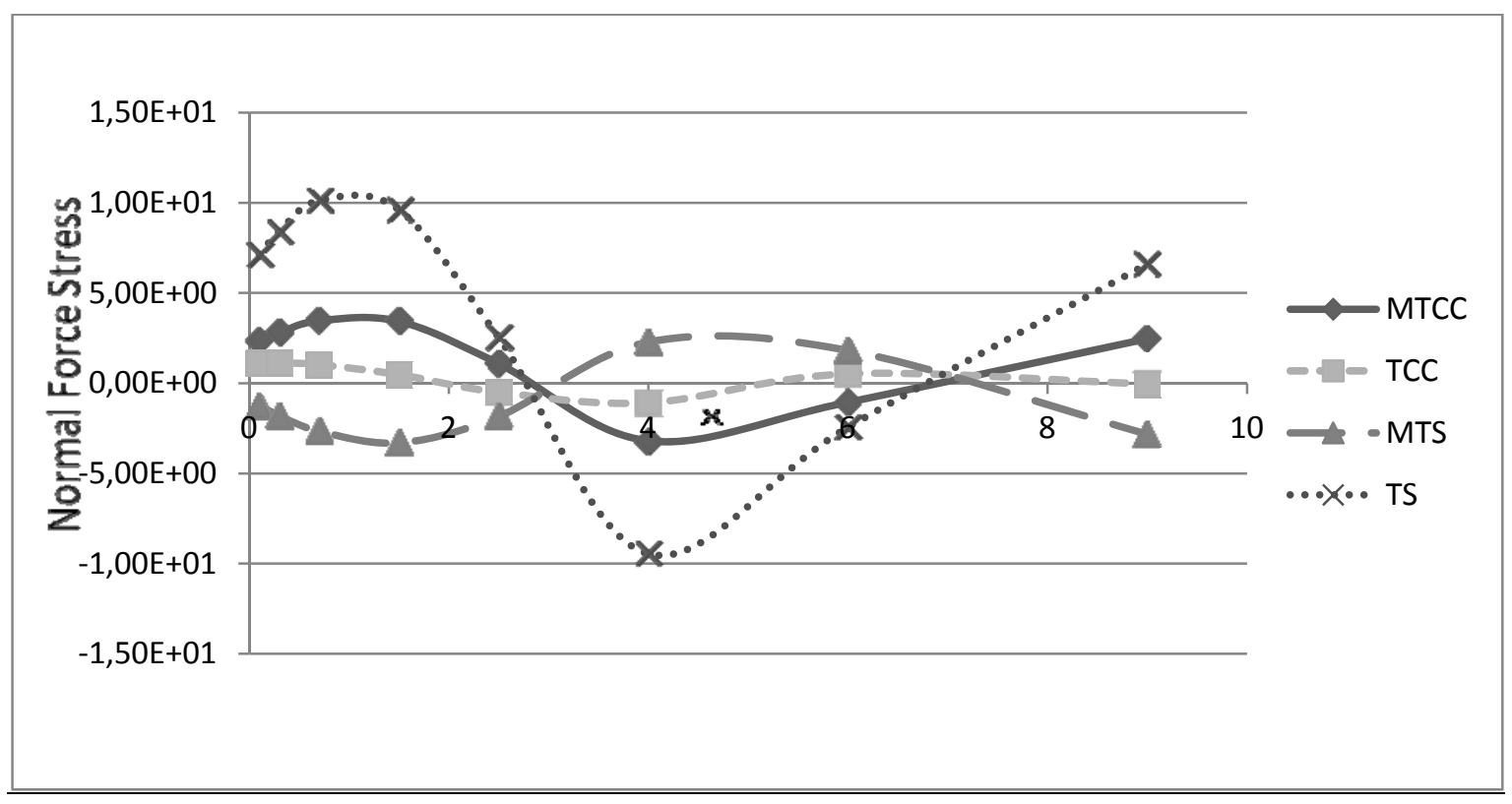

Fig.4. Variation of normal force stress with horizontal distance.

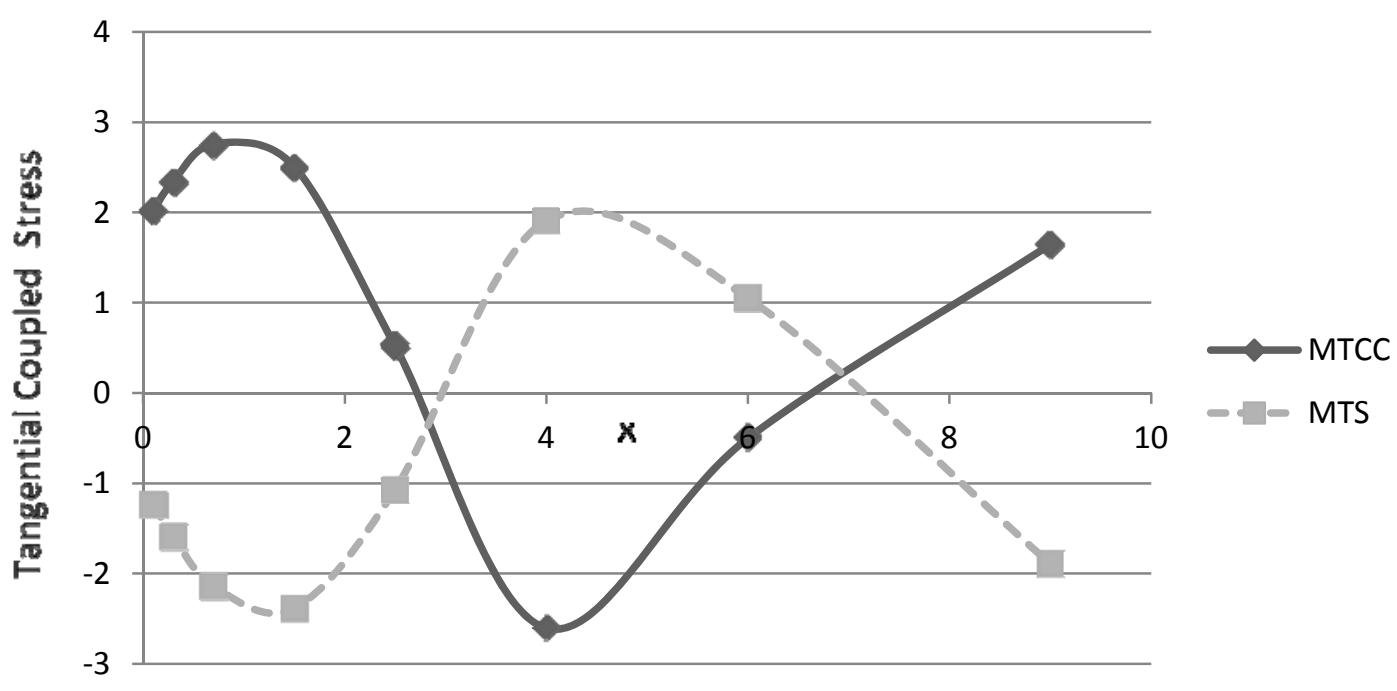

Fig.5. Variation of tangential couple stress with horizontal distance.

\section{Nomenclature}

\section{Medium-I}

$$
\begin{aligned}
A_{1}, A_{2}, A_{3}, & - \text { material constants } \\
A_{4}, B_{3} & \\
c_{I}^{*} & - \text { specific heat at constant strain } \\
j & - \text { microinertia } \\
K_{1}^{*} & - \text { coefficient of thermal conductivity }
\end{aligned}
$$


$m_{y z} \quad$ - tangential couple stress

$T_{1}$ - thermodynamic temperature

$\vec{u}^{I}$ - displacement vector

$\alpha_{t_{1}}$ - coefficient of linear thermal expansion

$\rho_{1}$ - density

$\sigma_{i j}^{I} \quad-$ stress tensor

$v_{1}$ - constitutive coefficient

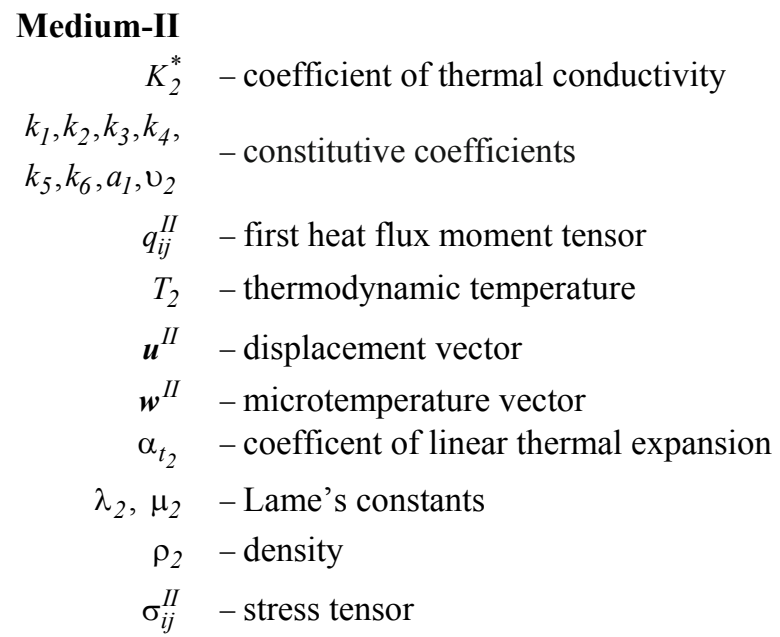

\section{References}

[1] Eringen A.C. and Suhubi E.S. (1964): Nonlinear theory of simple micro-elastic solids I. - International Journal of Engineering Science, vol.2, pp.189-203.

[2] Eringen A.C. and Suhubi E.S. (1964): Nonlinear theory of simple micro-elastic solids II. - International Journal of Engineering Science, vol.2, pp.389-404.

[3] Eringen A.C. (1965): Linear theory of micropolar elasticity. - ONR Technical report No. 29, School of Aeronautics, Aeronautics and Engineering Science, Purdue University.

[4] Eringen A.C. (1966): A unified theory of thermomechanical materials. - International Journal of Engineering Science, vol.4, pp.179-202.

[5] Eringen A.C. (1984): Plane waves in non-local micropolar elasticity. - International Journal of Engineering Science, vol.22, pp.1113-1121.

[6] Nowacki W. (1966): Couple stresses in the theory of thermoelasticity III. - Bulletin of the Polish Academy of Sciences Techanical Sciences, vol.8, pp.801-809.

[7] Eringen A.C. (1970): Foundation of micropolar thermoelasticity, Courses and Lectures. - No.23, CISM, Udine, Springer-Verlag, Vienna and New York.

[8] Tauchert T.R., Claus W.D. and Ariman T. (1968): The linear theory of micropolar thermoelasticity. - International Journal of Engineering Science, vol.6, pp.36-47.

[9] Tauchert T.R. (1971): Thermal stresses in micropolar elastic solids. - Acta Mechanica, vol.11, pp.155-169.

[10] Nowacki W. and Olszak W. (1974): Micropolar thermoelasticity. - in W.Nowacki and Olszak(eds.), Micropolar thermoelasticity, CISM Courses and Lectures, No.151, Udine, Springer-Verlag, Vienna.

[11] Dhaliwal R.S. and Singh A. (1987): Micropolar thermoelasticity. - Chapter 5, in R.B. Hetnarski(ed.), Thermal Stresses II, Mechanical and Mathematical Methods, ser. 2, North-Holland, Amsterdam. 
[12] Dhaliwal R.S. and Singh A. (1980): Dynamic Coupled Thermoelasticity. - Hindustan Publication Corporation, New Delhi, India 726.

[13] Eringen A.C and Kafadar C.B. (1976): Continum Physics. - New York: Academic Press, 4.

[14] Minagawa S., Arakawa K. and Yamada M. (1981): Dispersion curves for waves in a cubic micropolar medium with reference to estimations of the material constants for diamond. - Bulletin of the JSME, vol.24 (187), pp.2228.

[15] Kumar R. and Ailawalia P. (2006): Time harmonic sources at micropolar thermoelastic medium possessing cubic symmetry with one relaxation time. - European Journal of Mechanics A/Solids, vol.25, pp.271-282.

[16] Kumar R. and Ailawalia P. (2007): Moving load response in micropolar thermoelastic medium without energy dissipation possessing cubic symmetry. - International Journal of Solids and Structures, vol.44, pp.4068-4078.

[17] Kumar R. and Ailawalia P. (2007): Mechanical/thermal sources at thermoelastic micropolar medium without energy dissipation possessing cubic symmetry. - International Journal of Thermophysics, vol.28, No.1, pp.342-367.

[18] Kumar R. and Ailawalia P. (2008): Deformations in micropolar thermoelastic medium possessing cubic symmetry due to inclined loads. - Mechanics of Advanced Materials and Structures, vol.15, No.1, pp.64-76.

[19] Ailawalia P. and Kumar R. (2010): Time harmonic inclined load in micropolar thermoelastic medium possessing cubic symmetry with one relaxation time. - Tamkang Journal of Science and Engineering, vol.13, No.2, pp.117126.

[20] Othman M.I.A., Lotfy Kh. and Farouk R.M. (2009): Effects of magnetic field and inclined load in micropolar thermoelastic medium possessing cubic symmetry under three theories. - International Journal of Industrial Mathematics, vol.1, No.2, pp.87-104.

[21] Kumar R. and Partap G. (2010): Elastodynamic behavior of axisymmetric vibrations in micropolar thermoelastic cubic crystal plate. - Mechanics of Advanced Materials and Structures, vol.17, No.2, pp.99-107.

[22] Lotfy Kh. and Yahia N. (2013): Effect of magnetic field and a mode-I crack 3D problem in micropolar thermoelastic cubic medium possessing under three theories. - Journal of Solid Mechanics, vol.5, No.3, pp.253269.

[23] Abbas I.A., Kumar R. and Rani L. (2015): Thermoelastic interaction in a thermally conducting cubic crystal subjected to ramp-type heating. - Applied Mathematics and Computation 254, pp.360-369.

[24] Grot R.A. (1969): Thermodynamics of a continuum with microstructure. - International Journal of Engineering Science, vol.7, pp.801-814.

[25] Riha P. (1976) On the micro-continuum model of heat conduction in materials with inner struct. - International Journal of Engineering Science, vol.14, pp.529-535.

[26] Iesan D. and Quintanilla R. (2000): On a theory of thermoelasticity with microtemperatures. - Journal of Thermal Stresses, vol.23, pp.199-215.

[27] Iesan D. (2001): On a theory of micromorphic elastic solids with microtemperatures. - Journal of Thermal Stresses, vol.24, pp.737-752.

[28] Casas P.S. and Quintanilla R. (2005): Exponential stability in thermoelasticity with microtemperatures. International Journal of Engineering Science, vol.43, pp.33-47.

[29] Scalia A. and Svanadze M. (2006): On the representation of solutions of the theory of thermoelasticity with microtemperatures. - Journal of Thermal Stresses, vol.29, pp.849-863.

[30] Iesan D. (2006): Thermoelasticity of bodies with microstructure and microtemperatures. - International Journal of Solids and Structures, vol.43, pp.3414-3427.

[31] Aouadi M. (2008): Some theorems in the isotropic theory of microstretch thermoelasticity with microtemperatures. - Journal of Thermal Stresses, vol.31, pp.649-662.

[32] Iesan D. and Quintanilla R. (2009): On thermoelastic bodies with inner structure and microtemperatures. - Journal of Mathematical Analysis and Applications, vol.354, pp.12-23. 
[33] Scalia A., Svanadze M. and Tracinà R. (2010): Basic theorems in the equilibrium theory of thermo-elasticity with microtemperatures. - Journal of Thermal Stresses, vol.33, No.8, pp.721-753.

[34] Quintanilla R. (2011): On growth and continous dependence in thermoelasticity with microtemperatures. - Journal of Thermal Stresses, vol.34, No.9, pp.911-922.

[35] Steeb H., Singh J. and Tomar S.K. (2013): Time harmonic waves in thermoelastic material with microtemperatures. - Mechanics Research Communication, vol.48, pp.8-18.

[36] Chirita S., Ciarletta M. and D'Apice C. (2013): On the theory of thermoelasticity with microtemperatures. Journal of Mathematical Analysis and Applications, vol.397, pp.349-361.

[37] Kumar R. and Kaur M. (2014): Reflection and refraction of plane waves at the interface of an elastic solid and microstretch thermoelastic solid with microtemperatures. - Archives of Applied Mechanics, vol.84, pp.571-590.

[38] Ciarletta M., Passarella F and Tibullo V. (2015): Plane harmonic waves in strongly elliptic thermoelastic materials with microtemperatures. - Journal of Mathematical Analysis and Applications, vol.424, pp.1186-1197.

Received: March 15, 2016

Revised: October 13, 2016 